\title{
Vehicle Parking Assistance with Multi-Round Auction and Offload Reduction
}

\author{
Dr. S. Smys \\ Professor, \\ Department of Computer Science Engineering, \\ RVS technical Campus, \\ Coimbatore, \\ India. \\ Email: smys375@gmail.com
}

\author{
Dr. Subarna Shakya \\ Professor, \\ Department of Electronics and Computer Engineering, \\ Central Campus, Institute of Engineering, Pulchowk, \\ Tribhuvan University, \\ Pulchowk, Lalitpur, \\ Nepal. \\ Email: drss@ioe.edu.np.
}

\begin{abstract}
One of the most promising fields of vehicular fog computing (VFC) is by using it as a means to build a parking lot allotment system. Integrating this with parked vehicle assistance system, we can use VFC to its full potential. In this paper, we propose an automatic vehicle allocation system that guides the vehicles on the move to their allotted parking slot, making it simpler to park. Moreover, further add-ons such as monetary rewards are given means to compensate for the cost of service. The VFC is exploited to ensure that there is no delay in parking allotment. Moreover, the payment rule proposed by the system ensures budget balance, individual rationality and incentive compatibility. The performance output is measured using simulation results and it is observed that there is a significant improvement and the same is recorded.
\end{abstract}

Keywords: Multi-round Auction, Automatic vehicle parking, Parking assistance, Fog Computing;

\section{Introduction}

As our everyday life keeps changing due to increase in technological development IoT remains to be the key to the growth of information sector. A number of applications that are based on IoT include real-time online gaming, augmented reality, virtual reality, 5G services and fog computing have gained fame in the recent years because of their low latency services. However, along with their advantages, they also face a number of challenges in terms of security to be provided during fog computing. By implementing fog computing [1] in vehicles, vehicular fog computing approach is highly favoured in recent years because of low latency service. To meet the demands, the number of roadside units (RSU) which been introduced in various places of a locality are upgraded to provide computation and communication services based with fog servers. The only restriction with the RSUs is their limited density. In places where there are not enough RSUs, the vehicular ad hoc networks are implemented with parked vehicle assistance. It is worth mentioning that these parked vehicles can also be used to improve connectivity by means of exchanging and sharing of information with the surrounding vehicles [2].

However, as the number of vehicles being used by a household has increased, there is also increase in the need for parking spaces for each vehicle. In fact, survey shows that congestion during vehicle parking is one of the major causes of traffic in the streets. Moreover, searching for a parking slot will also result in unwanted waste of energy and time. To address these issues, a number of smart parking systems have been developed to decrease parking time, vehicle energy and fuel usage. It is also necessary to take into consideration several other parameters such as parking payment, walking cost and driving cost in order to efficiently utilize the parking allocation. In order to overcome these slight hiccups, the fog service provider can provide compensation as monetary rewards. In this paper a smart parking system that is integrated with virtual fog computing is proposed [3]. Similarly, we have also introduced monetary rewards to attract vehicles that are moving. The VFC system is evaluated using simulation using real time parking space deployment and street map. This paper is organised into 
Journal of Electrical Engineering and Automation (EEA) (2020)

Vol. 02/ No. 03

Pages: 129-134

https://www.irojournals.com/iroeea

DOI: https://doi.org/10.36548/jeea.2020.3.004

four sections. Section 2 gives a survey of recent work implemented in smart parking. Section 3 outlines the proposed methodology. The output of the proposed work is recorded in section 4 and a conclusion is drawn based on the outputs in section 5 .

\section{Literature Survey}

VFC has been one of the most prominent research topics in recent years. A number of low latency services have been introduced using VFC. In the beginning a software-defined network was used to build a VFC-based architecture which could be used to enhance and improve user-interfacing by many strides. In a simple VANET using VFC will face the difficulty of task offloading. To address this issues a predictive framework was proposed in [4] which had the ability to directly upload the tasks thereby saving time as well as utilization of extra resources. In [5] the author has addressed the issue of battery limitation in the vehicles. In their work, they proposed an allocation algorithm which could use the resources in a more efficient manner in order to address the issues of power control problem and join workload offloading. Similarly, they also used stochastic modeling at FNs and UEs to propose a more advanced solution that ensures higher accuracy. In [6] the research work indicates that if it is possible to influence a small scale of PVA vehicles, it will prove to be a greater aid to improving network connectivity. PVA vehicle are investigated using simulation, realistic survey and theoretical analysis.

In [7], [8] and [9] compact parking solutions have been proposed for parking of vehicles in a more efficient manner, taking economic interests into consideration. A complete survey of the smart parking systems that have been introduced over the years has resulted in proper classification of the parking system based on service dissemination, system deployment and information collection. Authors of [10-11] introduced a parking methodology that used uses mixed integer linear programming, taking into consideration optimal parking reservation and driver's cost function. In [12] a successful cloud-based parking architecture was introduced that incorporated IoT to manage and monitor the parking slots. A WSN (Zigbee) was used to implement it and the cloud operated as a data center. In this proposed work we have proposed an automatic vehicle allocation system that guides the vehicles on the move to their allotted parking slot, making it simpler to park [13].

\section{Proposed Work}

\subsection{Problem Formulation}

Fig.1. represents the proposed virtual fog computing based parking assistance system. Here let us consider $S H$ to represent ' $\mathrm{k}$ ' hotspots such that $S H=s_{1}, s_{2}, \ldots s_{k}$. Every hotspot is connected to many devices that are requesting multiple computation services. Consider $\mathrm{M}$ to be the number of parking places that are allocated for use by other people as parking slots. A simple parking sensor can be used to collect the availability of parking in real-time.

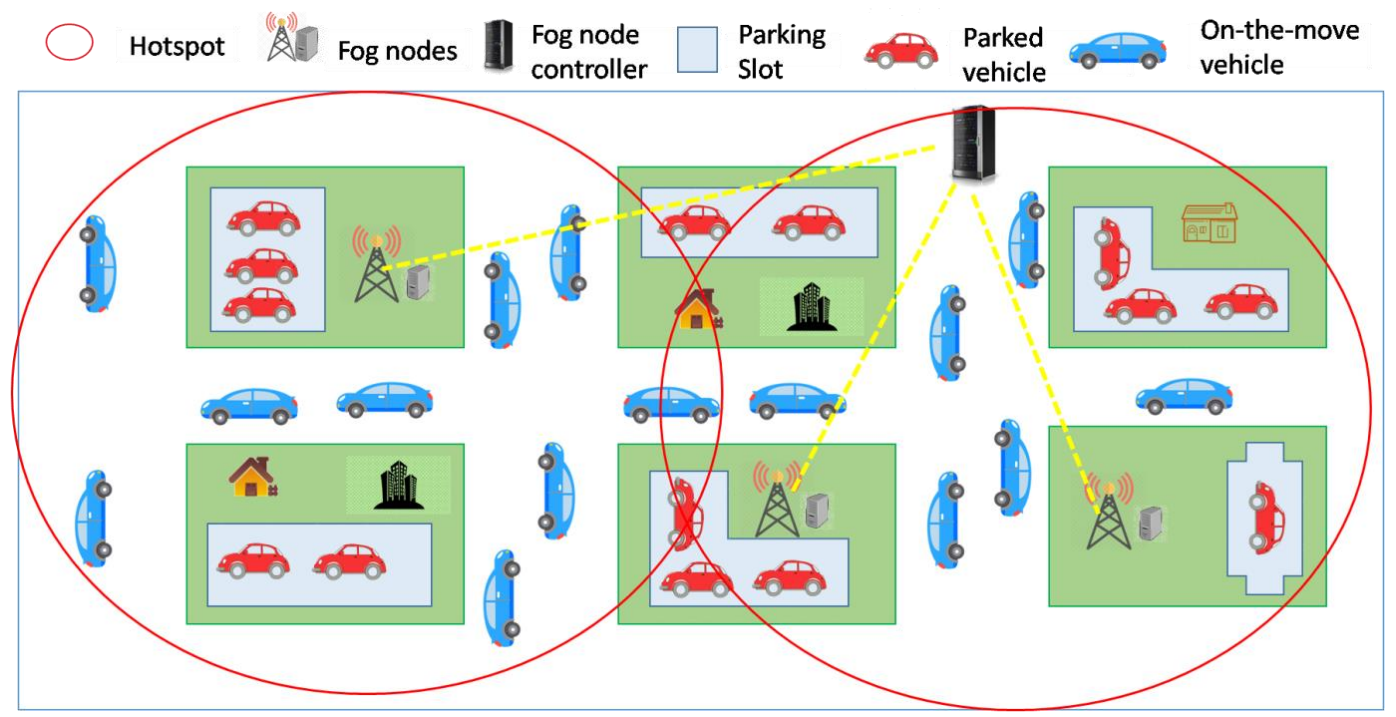

Fig.1. Smart Parking with Fog Computing 
Journal of Electrical Engineering and Automation (EEA) (2020)

Vol. 02/ No. 03

Pages: $129-134$

https://www.irojournals.com/iroeea

DOI: https://doi.org/10.36548/jeea.2020.3.004

\subsubsection{Parking}

The reservation state, departure event and arrival event of a vehicle are each represented by separate demotions to indicate criteria for parking such that:

$$
\begin{gathered}
a_{i, j}(t)= \begin{cases}1 & \text {, if vehicle } z_{i} \text { parks at spot } p_{j} \text { in the alloted time } t \\
0 & \text {, otherwise }\end{cases} \\
c_{i, j}(t)= \begin{cases}1 & , \text { if vehicle } z_{i} \text { parked at spot } p_{j} \text { will leave in the alloted time } t \\
0 & \text {, otherwise }\end{cases} \\
r_{i, j}(t)= \begin{cases}1 & \text {, if a parking slot } p_{j} \text { is reserved in the parking lot } z_{i} \text { the alloted time } t \\
0 & \text {, otherwise }\end{cases}
\end{gathered}
$$

where $a_{i, j}(t), c_{i, j}(t)$ and $r_{i, j}(t)$ represents the parking arrival, departure and reservation event respectively.

\subsubsection{Vehicles}

The overall parking capacity of the parking lot can be calculated using the formula:

$$
C_{j}(t)=C_{j}-\sum_{z_{i} \in B} \sum_{t^{\prime}}^{t-1} a_{i, j}\left(t^{\prime}\right)+\sum_{z_{i} \in B} \sum_{t^{\prime}}^{t-1} c_{i, j}\left(t^{\prime}\right)-\sum_{z_{i} \in B} r_{i, j}(t)
$$

To identify the parking location of a particular vehicle we can express it as :

$$
w_{i, j}= \begin{cases}1 & \text { when } z_{i} \text { is allocated to } p_{j} \\ 0 & \text { otherwise }\end{cases}
$$

Hence the vehicles that are on the move can be represented as $w_{i, j}$. For these vehicles, the parking slot is identified by measuring the distance between the current position of the vehicle and the allotted parking location which are denoted as $\varphi_{i}^{\text {dest }}$ and $\varphi_{i}^{\text {cur }}$.

\subsubsection{Hotspot}

The central geolocation serves as the hotspot of the system, represented by $\varphi_{k}^{h}$. Here the forward node (FN) is capable of determining the extent of requests in computing service from the end user. This load of work is calculated as

$$
\hat{\lambda}_{k}=\frac{\lambda_{k}}{m_{k}}
$$

where $\lambda_{k}$ is the workload arrival rate, $h_{k}$ is the service request and $m_{k}$ denotes the CPU numbers required based on delay and loading conditions. The use of FN ensures that cost-efficiency of the system is maintained by deleting redundant forward nodes.

\subsection{Single-Round Auction}

The vehicles that are in movement are provided with a reservation auction based on single-round multi-tem parking and use it to mainstream the parking slots available along with prior reservation spots. This will enable a non-delay parking mechanism. The Fog network and cloud computing (FNC) services announces the time slot for the parking vehicle and auctions it according to the offload price. A simple time sequence mechanism for the proposed parking reservation system is represented in Fig.2. 
Journal of Electrical Engineering and Automation (EEA) (2020)

Vol. 02/ No. 03

Pages: $129-134$

https://www.irojournals.com/iroeea

DOI: https://doi.org/10.36548/jeea.2020.3.004

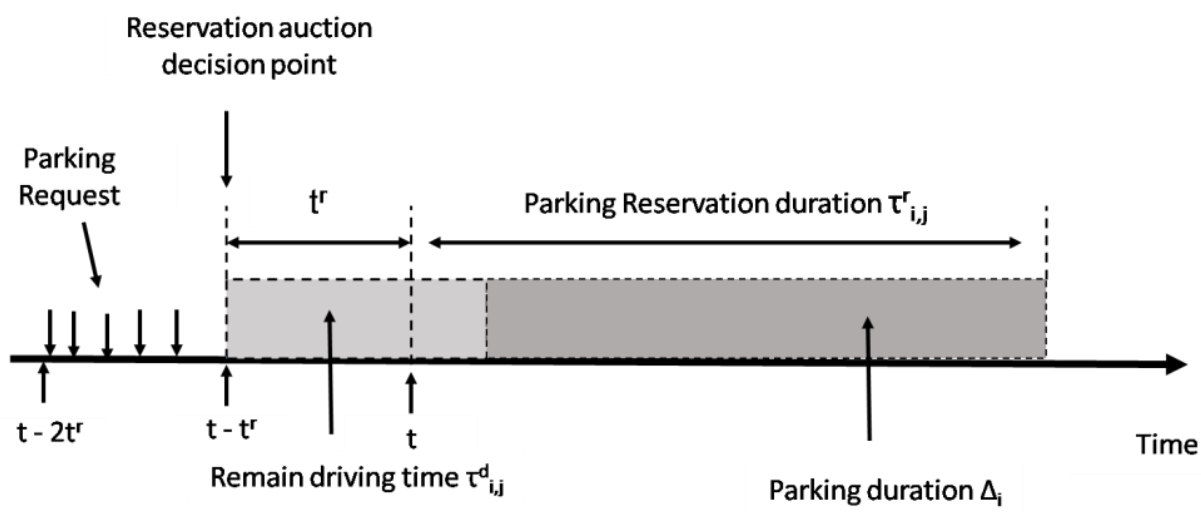

Fig.2. Parking Reservation based on Time-slot

\section{Results and Discussion}

The performance of the system is measured using the fixed offload price determined for hotspots and parking places, as announced in FNC. Fig.3 shows a comparison of the average vehicle utility based on offload price for single and greedy criteria. It is observed that at lower offload price, the fluctuation is considerably less due to the increase in CPUs allocated to aid the smart vehicle hotspots. As the offload price increases, there is also a significant increase in the average utility. This is further associated with the constraint in number of CPUs as shown in Fig.4.

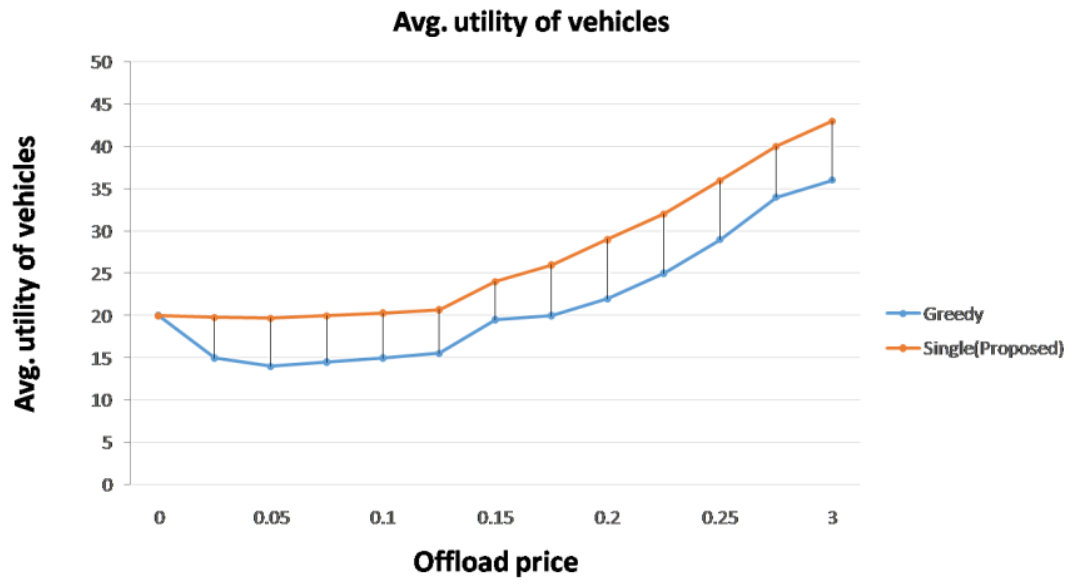

Fig.3. Average Vehicle Utility

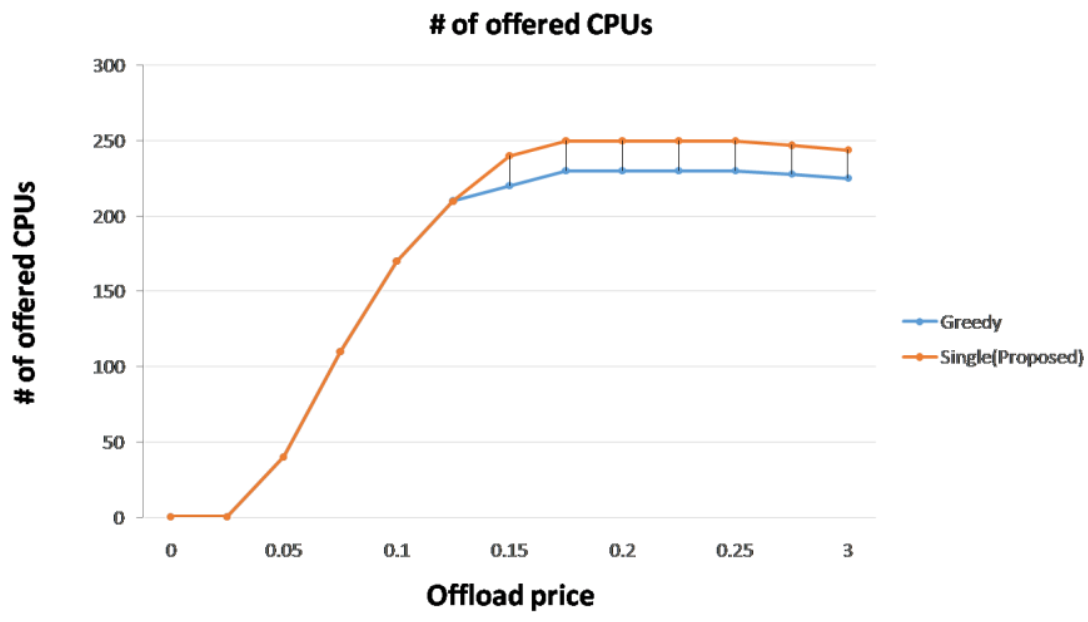

Fig.4. FNC profit 
Journal of Electrical Engineering and Automation (EEA) (2020)

Vol. 02/ No. 03

Pages: 129-134

https://www.irojournals.com/iroeea

DOI: https://doi.org/10.36548/jeea.2020.3.004

In terms of cost saving and service offloading, greedy is found to be behind single due to the increased CPUs in single. However, Fig.5 shows that when unnecessary offload payments occurs, there is no significant increase in FNC.

\section{Avg. utility of vehicles}

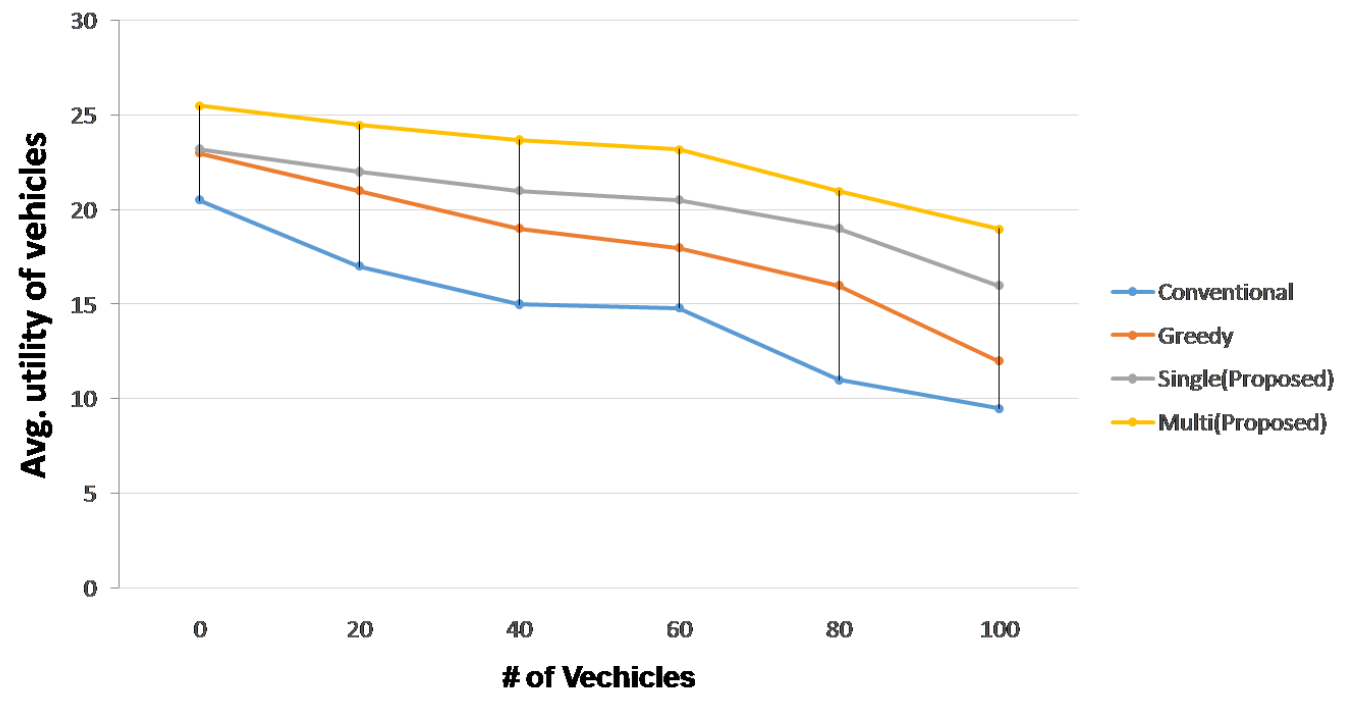

Fig.5

\section{Conclusion}

A Smart parking virtual assist system has been proposed in this work. The vehicles are guided to their allocated parking slots taking into account several factors such as parking time, vehicle energy and fuel usage. Using fog computing we have created an innovative smart parking methodology that uses optimal allocation and weight matching to determine the right spot for parking the vehicle. The results obtained satisfy the proposed auction design and further monetary incentive measures are used to encourage usage of this methodology. A multiround auction gives an optimal solution for parking places, smart vehicles and FNC.

\section{References}

[1] Tachibanada, Y., Mochizuki, T., Ishikawa, Y., Yoshida, S., \& Mukai, T. (2018). U.S. Patent No. 9,856,980. Washington, DC: U.S. Patent and Trademark Office.

[2] Zhang, Y., Wang, C. Y., \& Wei, H. Y. (2019). Parking reservation auction for parked vehicle assistance in vehicular fog computing. IEEE Transactions on Vehicular Technology, 68(4), 3126-3139.

[3] Sasajima, T., Aiga, Y., \& Asaba, T. (2013). U.S. Patent No. 8,560,169. Washington, DC: U.S. Patent and Trademark Office.

[4] Bindhu, V. (2020). An Enhanced Safety System for Auto Mode E-Vehicles through Mind Wave Feedback. Journal of Information Technology, 2(03), 144-150.

[5] Lin, I. C., Cheng, C. Y., \& Chen, H. Y. (2019). A real-time parking service with proxy re-encryption in vehicular cloud computing. Engineering Applications of Artificial Intelligence, 85, 208-213.

[6] Roy, A., Paul, J., Baidya, R., \& Devi, M. (2018). Parking places discovery and reservation using vehicular ad hoc networks. In Advances in Electronics, Communication and Computing (pp. 695-703). Springer, Singapore.

[7] Hakeem, A., Gehani, N., Ding, X., Curtmola, R., \& Borcea, C. (2019, November). Multi-destination vehicular route planning with parking and traffic constraints. In Proceedings of the 16th EAI International Conference on Mobile and Ubiquitous Systems: Computing, Networking and Services (pp. 298-307).

[8] Sungheetha, A., \& Sharma, R. (2020). Cost Effective Energy-Saving System in Parking Spots. Journal of Electronics, 2(01), 18-29.

[9] Sivaganesan, D. (2019). Efficient Routing Protocol with Collision Avoidance in Vehicular Networks. Journal of Ubiquitous Computing and Communication Technologies (UCCT), 1(02), 76-86.

[10] Mugunthan, S., \& Vijayakumar, T. (2019). Review on IoT based smart grid architecture implementations. j Electric Eng Autom, 1(1), 12-20. 
Journal of Electrical Engineering and Automation (EEA) (2020)

Vol. 02/ No. 03

Pages: $129-134$

https://www.irojournals.com/iroeea

DOI: https://doi.org/10.36548/jeea.2020.3.004

[11] Shirley, D. R. A., Ranjani, K., Arunachalam, G., \& Janeera, D. A. (2020). Automatic Distributed Gardening System Using Object Recognition and Visual Servoing. In Inventive Communication and Computational Technologies (pp. 359-369). Springer, Singapore.

[12] Kim, S., Jung, J., Kim, T., \& Min, H. (2018). A Resource Allocation Strategy for Cloud Computing in Vehicular Datacenter. The Journal of The Institute of Internet, Broadcasting and Communication, 18(4), 183-189.

[13] Pande, S. K., Panda, S. K., Das, S., Alazab, M., Sahoo, K. S., Luhach, A. K., \& Nayyar, A. (2020). A smart cloud service management algorithm for vehicular clouds. IEEE Transactions on Intelligent Transportation Systems. 